\title{
Erster Test für das Rote Kreuz
}

\section{Jürg Kesselring}

Mitglied des IKRK, Senior Botschafter und Neuroexperte, Kliniken Valens

\author{
2021 jährt sich die Internierung der französischen Bourbaki-Armee in der Schweiz \\ zum 150. Mal. Die damals gebotenen Hilfstätigkeiten legten unter anderem den \\ Grundstein für die Entwicklung des Schweizerischen Roten Kreuzes (SRK) und \\ machten die Grundsätze der Rotkreuzbewegung in ihrer praktischen Anwendung \\ bekannter.
}

Im Jahr 1871 kamen innerhalb von weniger als 72 Stunden 87847 Soldaten und Offiziere der französischen Armée de l'Est mit 12000 Pferden bei Les Verrières im Neuenburger Jura über die Grenze in die neutrale Schweiz. Die Armee, deren Personenstärke etwa 3\% der damaligen Schweizer Bevölkerung entsprach, war bis zu dessen kurz zuvor missglückten Selbstmordversuch unter dem Kommando von General Bourbaki gestanden und bewegte sich nun abgekämpft, schlecht ausgerüstet, ausgezehrt, frierend und krank, aber mit der Zustimmung des Bundesrats über die Schweizer Grenze. Die französische Armee hatte gegen die preussische Niederlage um Niederlage erlitten, sie war so schlecht ausgerüstet, schlecht trainiert und vor allem dramatisch schlecht geführt $[1,2]$. Dabei hatte doch Kriegsmarschall Le Bœuf dem Kaiser Napoleon III. noch am 15. Juli 1870 gemeldet: «Die Armee ist bereit. Es fehlt ihr nicht ein einziger Gamaschenknopf» («l'armée est prête, il ne lui manque pas un bouton de guêtre»).

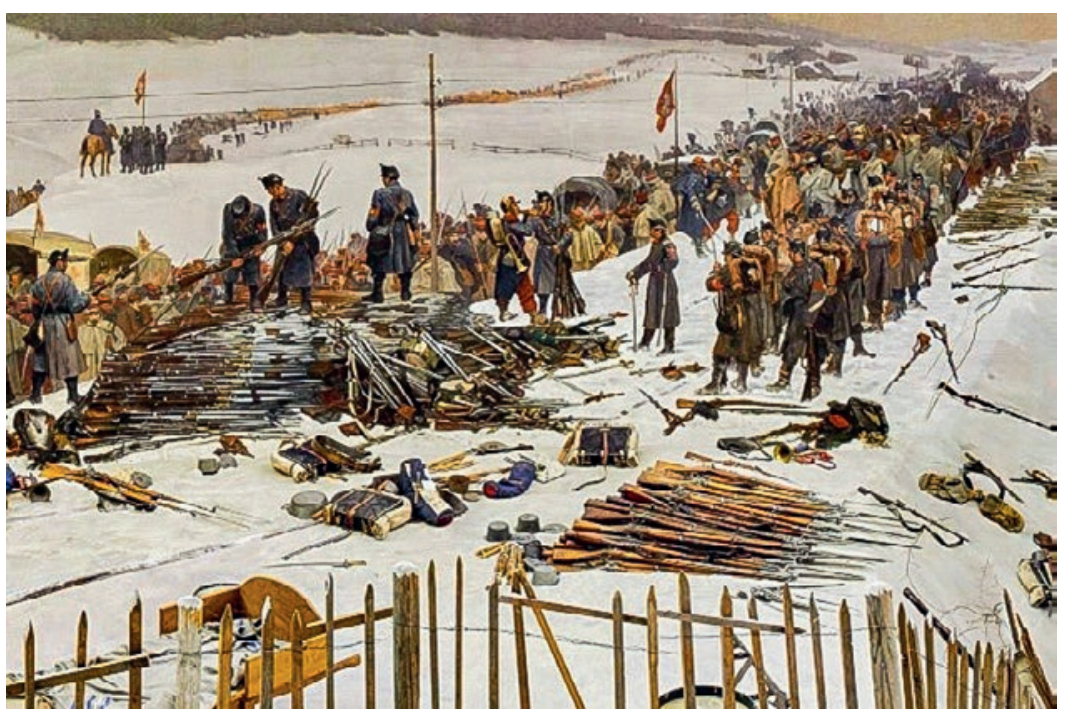

Die Internierten wurden in 188 Gemeinden zwischen Genf und Rorschacherberg untergebracht, verpflegt, medizinisch versorgt und falls nötig neu eingekleidet. Die Bevölkerung reagierte ausgesprochen hilfsbereit. Vielerorts wurde spontan Hilfe geleistet, und das Schweizerische Rote Kreuz (SRK) kam zu seinem ersten grossen Einsatz. Bei seiner Gründung 1866 war das SRK noch weitgehend unbekannt, fünf Jahre später wurde es zum Symbol eines grossen Zusammengehörigkeitsgefühls, das sich durch aktive Neutralität und Solidarität ausdrückt. Das SRK hatte bis dahin über wenig Handlungsmöglichkeiten und schwache kantonale Strukturen verfügt, nach dem Kriegsausbruch 1870 aber bildeten sich zwanzig lokale, sehr aktive Sektionen.

Ein Augenzeuge aus einem Thuner Bataillon hielt fest: «Der Einmarsch begann, ein Bild des Entsetzens, der schauerlichen Unordnung und Zerfahrenheit einer Armee im Rückzug ... ohne Murren, willenlos, gebrochen warfen sie ihre Waffen zur Erde, ganze Berge von Waffen aller Art erhoben sich entlang der Strasse, durch die diese zerfetzte Armee zog. Tote und sterbende Pferde zeichneten ihren Weg. Zwei ganze Tage und eine Nacht sahen wir diese traurigen Armeetrümmer an uns vorbeiziehen und noch kommen fort und fort ganze Schwärme von Nachzüglern» (Augenzeugenbericht von Hans Frutiger vom 1.2.1871 im Bourbaki Panorama).

\section{Herzlicher Empfang durch die Schweizer Bevölkerung}

Armee und Behörden waren bei dieser grössten Flüchtlingsaufnahme in der Schweizer Geschichte auf das Mitwirken der humanitären Organisation und der Bevölkerung angewiesen. In einem zeitgenössischen Bericht aus dem Kanton Freiburg heisst es: «Die Militärverwaltung konnte lediglich Brot - und manchmal Wein - an die zu Fuss oder mit der Eisenbahn ankom- 
menden oder wieder abreisenden Soldatenströme verteilen. Den Rest leisteten Privatpersonen durch ihr wohltätiges Engagement. Ein Ruf des öffentlichen Ausrufers zu jeder beliebigen Uhrzeit genügte und schon wurden massenhaft Suppen und Hilfe jeglicher Art bereitgestellt.» Zusätzliche Schwierigkeiten bereitete das Auftreten der "Blattern-Krankheit» (Pocken), «dass die Betreffenden die sanitätspolizeilichen Anordnungen gehörig berücksichtigen mögen. Es handelt sich um das Wohl Aller und der betreffende Arzt und die Behörden tun gewiss besser, wenn sie eher etwas zu strengere Massnahmen ergreifen, besonders im Entstehen einer solchen Calamität» (was einem in Corona-Zeiten bekannt vorkommt). In den französischen Truppen grassierten die Pocken, und mit den herumreisenden Soldaten erreichten sie alle umliegenden Länder. Eine halbe Million Tote und verschärfte Impfgesetze waren die Folge [3]. Der Krieg hatte aber auch gezeigt, dass im Gegensatz zu den französischen Soldaten die deutschen grösstenteils von der Krankheit verschont geblieben waren: Im deutschen Militär bestand Impfpflicht, im französischen nicht.

Die erfolgreiche Zusammenarbeit in der Rotkreuzbewegung mit lokalen Freiwilligen dauert bis heute an: Ohne diese Unterstützung wäre sie nicht in der Lage, die nationalen und internationalen Herausforderungen zu meistern. Die heute aktuelle Frage stellte sich bereits damals: Wird das kleine Land die plötzliche Flüchtlingskrise bewältigen können? Ein Korrespondent der NZZ schrieb am 7. Februar 1871: «Seit drei Tagen bietet sich unsern Augen das schreckliche Schauspiel einer Armee in Deroute. Die Mannschaft, schwarz von Pulver und Schmutz, sieht kaum kräftiger aus als ihre Pferde. Die Bevölkerung der Dörfer im Val-deTravers verrichtet Wunder der Hingebung und entblösst sich von Allem, um dieses Uebermass von Elend zu erleichtern. Aber ihre Hingebung findet ihre Grenze in den vorhandenen Mitteln, und diese Grenze ist erreicht.»

\section{Die heute aktuelle Frage stellte sich bereits damals: Wird das kleine Land die plötzliche Flüchtlingskrise bewältigen können?}

Die Truppen in ihrem schrecklichen Zustand, gezeichnet von Strapazen, Kälte und Hunger, wurden untergebracht, wo gerade Platz war - in Kasernen in Aussenquartieren, Remisen der Post, einer Brauerei, Galerien, Schulhäusern oder Bibliotheken, in Kirchen und Kapellen: «Improvisationsfreudig wird daher auch noch die barocke Jesuitenkirche genutzt. Mit zwei Feuerstellen im Innern der Kirche werden die weiteren 1100 Männer gewärmt», heisst es. Für die Bevölkerung

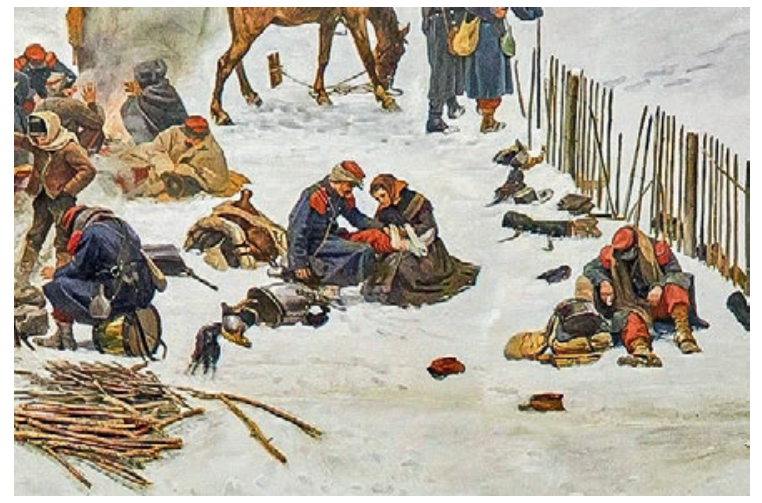

waren die Internierten gewissermassen auch eine Sensation: In vielen ländlichen Gemeinden hatten die Menschen noch kaum Fremde gesehen, geschweige denn Menschen mit dunkler Hautfarbe. Für Frankreich kämpften auch Soldaten aus Nordafrika (die von der deutschen Kriegspropaganda durchweg als «Menschenfresser» bezeichnet wurden). Die anfängliche Zurückhaltung war allerdings nur von kurzer Dauer. Schon bald dominierten Mitgefühl und Solidarität für die von Krieg, Kälte und Krankheit gezeichneten Soldaten [4]. Der Empfang durch die Schweizer Bevölkerung war herzlich. Die Internierten wurden gepflegt und betreut. Trotzdem starben rund 1700 Soldaten, woran heute noch mehrere Grabmäler erinnern.

\section{Die Schweiz als Kriegsschauplatz?}

Oberstdivisionär Eugen Bircher schrieb während des Zweiten Weltkrieges über die Ereignisse bei der Internierung der Bourbaki-Armee in der Helvetischen Militärzeitschrift [5]: «Nun jährt es sich zum siebzigsten Male in unvergleichlich schwerer Zeit, dass an unserer Nordwestgrenze das geschlagene französische Heer unter General Clinchant entwaffnet in die Schweiz einrückte. Nicht eindrucksvoll genug kann man unserem Volke jene bewegten Ereignisse vor Augen führen; stand es doch auf der Messerschneide, dass infolge mangelhafter und ungenügender Vorsorge der politischen Behörden es beinahe zum Einmarsch der Truppen beider Parteien auf unser Gebiet gekommen wäre. Dank allein der kaltblütigen Energie von General Hans Herzog war es noch in letzter Stunde möglich, durch ein gütiges Schicksal begünstigt, die gefahrdrohende Lage zu meistern.» Ein Veteran wird in diesem Bericht zitiert: «Es kam vor, dass Bataillone hungrig, durstig, müde, halberfroren am ursprünglichen Bestimmungsort angekommen, eine Depesche vorfanden mit der Einladung, den Marsch noch einige Stunden fortzusetzen. 'Sack auf! Vorwärts marsch!' Da durchfurchte denn hie und da ein Zürcher Stossseufzer die grimmig 
kalte Winternacht. Wenn die Schweizer Soldaten dann aber zu ihrer Beschämung sahen, wie die welschen Waffenbrüder mit ihrem Spruche 'à la guerre comme à la guerre' alles Unangenehme entschuldigten, da zogen die Hungernden die Patronentaschen-Riemen enger und beruhigten so wenigstens den knurrenden Magen, so gut er's eben glauben mochte. Es stand auf Messers Schneide, ob unser Land zum Kriegsschauplatz werden sollte oder nicht.»

Am 1. Februar, frühmorgens um 3 Uhr, nachdem General Herzog am Vorabend auf einem Güterzug spät nach Verrières gelangt war, trafen bei ihm der französische Konsul von Neuenburg mit Oberstleutnant Chevals ein. Sie verlangten die Bewilligung für den Übertritt der Bourbaki-Armee auf Schweizer Boden, nachdem die Befehle dazu von französischer Seite schon vorher gegeben worden waren. Herzog diktierte um 4 Uhr morgens den Vertrag, wonach der Übertritt erst nach vollständiger Entwaffnung gestattet wurde. In dem Zollhäuschen an der Grenze unterzeichnete General Clinchant den Vertrag. Sofort begann der Übertritt.

\section{Neutralität und Solidarität}

Ein Hilfskomitee grösserer Dimension bestand schon seit 1863 in Form des «Comité International de Secours aux Militaires Blessés» (Internationales Komitee für Kriegsverletzte), das spätere Rote Kreuz. Es wurde auf Initiative von Henry Dunant in Genf gegründet.

1864 lud der schweizerische Bundesrat 15 europäische Staaten und die USA zu einer Konferenz nach Genf ein. Daraus resultierte ein völkerrechtliches Abkommen zum Schutz (Neutralisation) der Verwundeten und Sanitäter im Konfliktfall, 1. Genfer Konvention genannt. Seit 1875 trägt die Organisation den Namen «Internationales Komitee vom Roten Kreuz» (IKRK). Die Rotkreuzbewegung stützt sich auf Grundprinzipien und verpflichtet sich zu deren Umsetzung: Humanität, Unparteilichkeit, Neutralität, Unabhängigkeit [6], Freiwilligendienst, Einheit, Universalität auf der Grundlage des Humanitären Völkerrechtes [7]. Neutralität war schon in den Gründerzeiten von Henry Dunant und seinen Kollegen im Komitee (General Guillaume-Henri Dufour, Gustave Moynier, Louis Appia, Théodore Maunoir) ein umstrittener Begriff, weil er ja sprachlich korrekt eigentlich bedeuten würde: ne utrum = «keines von beiden», er aber in der Praxis eben «beide» meint.

Die Neutralität war und ist ein zentrales Element der schweizerischen Aussen- und Sicherheitspolitik. Historisch und verfassungsrechtlich war die Neutralität aber nie ein Ziel unseres Staatswesens an sich, sondern ein Mittel unter mehreren zur Verwirklichung der eigentlichen, zentralen Ziele, nämlich insbesondere der Aufrechterhaltung einer möglichst grossen staatlichen Unabhängigkeit. Neutralität bedeutet im Völkerrecht die Nichtteilnahme eines Staates an Kriegen zwischen anderen Staaten. Die Schweiz konnte sich dadurch auf ihre Innenpolitik konzentrieren. Dies trug auch indirekt zum Auf- und Ausbau der direkten Demokratie, des Föderalismus, des Wohlstandes und

Mit «à la guerre comme à la guerre» wurde Unangenehmes entschuldigt.

der auf dem Milizprinzip beruhenden Verteidigungsarmee bei. Die Schweiz hat Neutralität in ihrer Geschichte nicht als ein starres, ein für alle Mal fixiertes Institut aufgefasst, sondern immer wieder flexibel den internationalen Notwendigkeiten und den eigenen Interessen angepasst [8].

Um die Grundprinzipien des IKRK bei allen RotkreuzAktionen umzusetzen (siehe www.icrc.org), wie es auch heute (leider) immer noch notwendig ist, dienen folgende Stichworte, die auch für friedliche Auseinandersetzungen gültig und empfehlenswert sind [1]: Empathie, aktives Zuhören, kritisches Denken, keine Vorverurteilung, nicht-verletzende Kommunikation, kollaborative, vermittelnde Verhandlungen, persönliche Resilienz, innerer Frieden [9].

In Luzern erinnert seit 1889 das eindrückliche Bourbaki-Panorama und in Schaffhausen neu eine Ausstellung im Zeughaus Museum im Zeughaus (Bourbaki und Schaffhausen) an die damaligen für die Schweiz identitätsbildenden Ereignisse.

www.bourbakipanorama.ch

\section{Bildnachweis}

Edouard Castres, Zug der Verwundeten (Studie zum Bourbaki-Panorama), 1876/1877. www.bourbakipanorama.ch

\section{Literatur}

1 Bugnion François. The arrival of Bourbaki's army at Les Verrières. The internment of the First French Army in Switzerland on 1 February 1871. In: Int Rev Red Cross. 1996;311:181-93.

2 Bugnion François. Il y a 150 ans, l'arrivée de milliers de «Bourbaki» aux Verrières, une expérience humanitaire fondatrice. In: Le Temps. $1^{\text {er }}$ février 2021.

3 Mäder Claudia. Impfen ist von Vorteil. Das galt zumindest im Krieg zwischen Frankreich und Preussen. In: NZZ, 1.2.2021.

4 Schwarzenbach Robin. Vor 150 Jahren - Die Bourbakis kommen! In: NZZ, 30.1.2021.

5 Bircher Eugen. Vor 70 Jahren. Zum Uebertritt der Bourbaki-Armee in die Schweiz am 1. und 2. Februar 1871. In: Helvetische Militärzeit schrift 107 (2/87), Organ der Schweizerischen Offiziersgesellschaft.

6 Thürer Daniel. Dunant's pyramid: thoughts on the «humanitarian space». In: Int Rev Red Cross. 2007;89:47-61.

7 Kellenberger Jakob. Humanitäres Völkerrecht. Frauenfeld: Huber; 2010.

8 www.geschichte.redcross.ch/ereignisse/ereignis/internierungder-bourbaki-armee-in-der-schweiz.html [abgerufen 24.5.21].

9 Kesselring Jürg. Teilen statt an sich raffen. In: moneta. 2015;4:16. 